\title{
El índice de pobreza hídrica para México: una comparación con países de la OECD
}

\section{The water poverty index for Mexico: a comparison with OECD countries}

\author{
M. J. Olivas y M. Camberos \\ Recibido: diciembre 14 de 2020 - Aceptado: junio 30 de 2021
}

\begin{abstract}
Resumen - Ante la preocupación por la escasez de agua en el mundo y la persistencia de bajos índices de desarrollo y altos de marginación, pobreza, desigualdad y deterioro ambiental, el propósito de esta investigación es conocer la situación que guarda México en la pobreza hídrica (PH), para lo cual se diseña una estrategia de cálculo del Índice de Pobreza Hídrica (IPH), acotado entre 0 máxima y 100 menor pobreza, el cual se compara con una muestra no probabilística de países pertenecientes a la Organización para la Cooperación y Desarrollo Económico (OCDE), a la cual pertenece México. Los países incluidos en el estudio se seleccionan bajo el criterio de existencia de los indicadores elegidos para la estimación de la PH. EI IPH se estima con el método de índice compuesto y balanceado estadísticamente con el Análisis de Componentes Principales (PCA) para detectar la correlación entre sus variables de agregación y así evitar su doble conteo. Como resultado de la estrategia de cálculo del IPH se halló innecesaria la eliminación de alguna variable y se conservaron los cinco indicadores que lo componen: Recursos, Uso, Acceso, Capacidad, y Medio Ambiente. La estimación mostró que el IPH de México fue el menor (mayor grado de $\mathbf{P H}$ ) comparado con el observado en los países en estudio, en concordancia con el rezago del país respecto a factores socioeconómicos, lo cual confirma la necesidad de la atención integral a la problemática del agua, para evitar la incompleta visión que representa la sola medida de cobertura de servicios de agua y saneamiento.
\end{abstract}

Palabras clave - políticas del agua, indicador compuesto, cinco

\footnotetext{
${ }^{1}$ Producto derivado de los proyectos de investigación "Pobreza de agua en México y sus regiones hidrológico-administrativas 2015: evaluación mediante el índice de pobreza hídrica" en el Centro de Investigación en Alimentación y Desarrollo A. C.

M. J. Olivas, Centro de Investigación en Alimentación y Desarrollo, A C., Hermosillo, Sonora, México, email: juliana.olivas@estudiantes.ciad.mx.

M. Camberos, Centro de Investigación en Alimentación y Desarrollo, A. C., Hermosillo, Sonora, México, email: mcamberos@ciad.mx.
}

Cómo citar este artículo: Olivas, M. J. y Camberos, M. El índice de pobreza hídrica para México: una comparación con países de la OECD, Entre Ciencia e Ingeniería, vol. 15, no. 29, pp. 54-62, enero-junio 2021. DOI: https://doi.org/10.31908/19098367.1848.

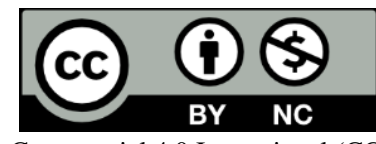

Attribution-NonCommercial 4.0 Intenational (CC By-NC 4.0) dimensiones, análisis de componentes principales.

Abstract - Motivated by concerns about water scarcity in the world and the persistence of low rates of development and high levels of marginalization, poverty, inequality and environmental deterioration, the purpose of this research is to probe the water poverty (WP) of Mexico, by means of implementing a procedure to calculate the Water Poverty Index (WPI), ranging from 0 as maximum and 100 as least poverty, and compare it with a nonprobabilistic sample of countries belonging to the Organization for Economic Cooperation and Development (OECD), of which Mexico is a member. The countries included in the study are selected under the criterion of existence of the indicators chosen to estimate WP. The WPI is estimated using the composite index method, statistically balanced with the Principal Component Analysis (PCA) to detect the correlation between its aggregated variables to avoid double accounting. As a result of the calculation procedure, any elimination of variables was found unnecessary and the five indicators, Resources, Use, Access, Capacity, and Environment, were maintained. The estimation showed that the WPI in Mexico was the lowest (higher WP) compared to that observed in the sample of countries, in accordance with the country's lag regarding socioeconomic factors, findings that confirm the need for comprehensive attention to the water issues in order to avoid the incomplete vision that represents the sole measuring of coverage of water and sanitation services.

Keywords - water policies, composite index, five dimensions, principal component analysis.

\section{INTRODUCCIÓN}

La carencia o escasez en relación con el agua, su uso y distribución eficiente, la disponibilidad y acceso, son un tema que ha venido cobrando fuerza en las sociedades modernas, debido a que afecta todas las esferas del desarrollo; esto es, la dimensión humana, social, económica y ambiental; dimensiones que Sen [1] eleva al grado de libertades fundamentales "que permiten a los individuos satisfacer el hambre, conseguir un nivel de nutrición suficiente, poner remedio a enfermedades tratables, vestir dignamente, tener una vivienda aceptable, y disponer de agua limpia y de servicios de saneamiento". Es así que se ha conceptualizado la 
Pobreza Hídrica (PH) como "una situación en la que una nación o región no puede pagar el costo del agua limpia sostenible para todas las personas en todo momento" [2].

Por otro lado, poner fin a la pobreza en general es el Objetivo Uno del Desarrollo Sostenible (ODS) de la agenda 2030 de la Organización de las Naciones Unidas [3], y proveer de agua y saneamiento seguros ${ }^{2}$ es el Objetivo 6, cuyas metas 6.1 y 6.2 son mucho más ambiciosas que la meta 7c de los Objetivos del Milenio (ODM), que es reducir a la mitad la proporción de la población sin acceso a agua potable y saneamiento, meta superada por México. Cabe recordar que México está comprometido a cumplir con los ODS, así como lo estuvo con los (ODM).

Es conocido que muchos países carecen de la disponibilidad de volúmenes de agua renovable per cápita suficientes para satisfacer las necesidades básicas, como lo muestran los datos de AQUASTAT [4] y algunos países pertenecientes a la Organización para la Cooperación y el Desarrollo Económico (OCDE) no son la excepción; es pertinente mencionar que México pertenece a la OCDE desde mayo de 1994. En un claro contraste, para países con abundantes recursos renovables, el agua también se ha convertido en un reto que depende de varios factores, no sólo de la disponibilidad y cobertura de agua, sino también del ingreso y la posición socioeconómica de los hogares. En consecuencia, el problema se refiere a la dificultad que tienen los gobiernos de los países para suministrar agua y saneamiento seguros a toda su población.

En este contexto, es pertinente precisar que uno de los indicadores más utilizados para medir la escasez de agua es el de Falkenmark [5], que se refiere a la Disponibilidad de Agua Renovable per cápita/año en $\mathrm{m}^{3}$, bajo los siguientes criterios: 1) Sin estrés valores > a 1700, 2), Con estrés < 1700, pero > $1000,3)$ Con escasez valores $<1000$, y 4) con escasez absoluta valores $<500$. De acuerdo con este indicador la mayor disponibilidad la presenta Islandia, la menor corresponde a la República Checa y solo cuatro países presentan un problema de escasez hídrica: Bélgica, República Checa, Dinamarca y Polonia; mientras que México no muestra ningún estrés, según este criterio [4].

Con todo y este panorama respecto al agua que presentan los países de la OCDE, si bien no preocupante, en algunos de los países miembros como México, la gestión efectiva y eficiente del agua sigue siendo una prioridad, ya que la presión sobre los recursos hídricos continúa aumentando. Con la mirada puesta en el año 2050, la OCDE [6] pág.21 señala que habrá una gran competencia por los mismos, en parte debido al crecimiento demográfico y económico, por lo que se prevé disminución de la calidad del agua, necesidad constante de ampliar la cobertura de agua potable y saneamiento; un deterioro mayor en el abastecimiento de aguas subterráneas; sin dejar de observar la amenaza que representa el cambio climático.

\footnotetext{
${ }^{2}$ Agua segura: Disponible en la vivienda en forma continua, asequible y libre de contaminación. Saneamiento seguro: Inodoro en la vivienda, y eliminación de excretas en el sitio, o tratadas fuera.
}

Al 2015, en México, la disponibilidad anual de agua renovable per cápita es de $3656 \mathrm{~m}^{3}$, la cobertura nacional de suministro de agua potable es de $95 \%$ y el saneamiento es de 92\% [7] pág.113; datos que no son muy diferentes a los de algunos países de la OCDE del presente estudio; como sí lo son las desigualdades sociales, económicas, y ambientales existentes en México con respecto a esos países; dado que México está rezagado, tal como lo muestran los indicadores de bienestar "The Better Life Index" [8] para las dimensiones de ingresos y educación, donde ocupa el último lugar, y en la dimensión de ambiente el penúltimo lugar, antes de Grecia, además de que en salud se encuentra entre los tres más bajos, antes de Japón y Polonia. Ello hace suponer que, a pesar de la alta cobertura de agua y saneamiento, la PH de México es alta (IPH bajo) comparada con la de los países seleccionados en este estudio.

En este marco de análisis es pertinente preguntarse ¿Cuál es la estrategia de cálculo más adecuada para medir de la PH?, ¿Cuál es el grado de PH para México?, ¿Cuál es la posición del IPH para México en comparación con los países de la OCDE?, ¿Cuál es el indicador que tiene mayor influencia en el IPH para México y para cada uno de los países de la OCDE seleccionados?, ¿Existe correlación entre el IPH y la pobreza de ingresos?; preguntas que trataremos de responder a lo largo de esta investigación.

Considerando que las mediciones oficiales de la cobertura y disponibilidad de agua enmascaran la situación real de la misma, y no contribuyen a hacer visible el problema, el propósito de esta investigación es indagar por medio del IPH el grado de la $\mathrm{PH}$ en México, en comparación con la de algunos países de la OCDE, lo cual se logrará con la consecución de los siguientes objetivos específicos: proponer e implementar una estrategia de cálculo para el IPH de México y de una muestra de países de la OCDE, así como analizar la influencia de los indicadores que más contribuyen a la $\mathrm{PH}$ de México, e indagar el grado de correlación entre el IPH y la pobreza de ingresos en la muestra de países.

La hipótesis planteada es que a pesar de la alta cobertura de agua potable y saneamiento que presentan los informes oficiales, la $\mathrm{PH}$ en México es alta, comparada con la observada en los países de la OCDE seleccionados para este estudio, y cuando son considerados factores socioeconómicos resulta más evidente.

\section{A. Revisión conceptual y empírica de la pobreza hídrica}

Una revisión del concepto de pobreza pone de manifiesto diversas acepciones en el transcurso del tiempo; sin embargo, pese a la posible controversia en su definición, existe coincidencia en relacionarla con una condición de carencia o escasez evaluadas objetivamente [9] pág. 31. Bajo esta premisa, dependiendo del tipo de carencia, se ha conceptualizado a la pobreza como de ingresos, de capacidades y de necesidades básicas [1], también como alimentaria, energética, hídrica, entre otras; todas ellas aceptadas por la comunidad científica y académica, sobre la base del enfoque del aumento del bienestar [10].

En investigaciones posteriores, Molle y Mollinga [11] 
clasifican los usos fundamentales del agua en U1-potable, U2doméstica, U3-producción, U4-económica y U5-necesidades ambientales y los combinan en una matriz con los tipos de escasez (S1-física, S2-económica, S3-de gestión, institucional, y S4-política). Por ejemplo, una de estas combinaciones sería U1S2, cuya lectura es: el agua potable para consumo humano está disponible pero una persona no puede pagarla. Los autores concluyen que el nexo agua-sociedad no puede ni debe reducirse solamente a los medios técnicos y financieros para suministrar cualquier cantidad de agua que se solicite.

Considerando que la escasez del agua es un problema a nivel global, Rijsberman [12] escribe acerca de la dificultad de determinar si el agua es realmente escasa en el sentido físico a escala mundial, o si se trata de un problema de suministro, o si bien, estando disponible se trata de un problema de demanda. Por otro lado, puntualiza el autor, "que la extracción de agua para uso doméstico, alimentario e industrial tiene un gran impacto en los ecosistemas en muchas partes del mundo, inclusive, en aquellos países que no se consideran con escasez de agua".

Lo anterior está en una clara concordancia con lo expresado por Lissner, Sullivan, Reusser y Kropp [13], quienes afirman que para tomar decisiones informadas con respecto al agua, se requieren enfoques integrados que permitan ver los determinantes en su conjunto, y ofrecen su visión desde dos perspectivas: un enfoque para medir las condiciones de vida humana, adecuadas para el bienestar y el desarrollo y un segundo enfoque que incluye aspectos de cantidad, calidad, y acceso de manera integrada al agua. En ese sentido Marvin [14] hace énfasis en la amenaza que el problema de la $\mathrm{PH}$ representa para la población, especialmente para los enfermos, y recalca el hecho de que no se puede suponer que por existir redes de infraestructura urbana se debe dar por sentado el acceso equitativo a los servicios públicos; lo cual queda bastante claro, ya que estos servicios, incluido el suministro de agua, están comúnmente insertos en la dinámica del mercado, en la cual a veces solo es posible obtenerlos si se tienen ingresos.

En el mismo contexto, la literatura en el tema muestra que simultáneamente se ha creado una nueva conceptualización, medición y evaluación de la PH, conocer solo el dato de cuánta agua renovable está disponible para un determinado número de personas no proporciona una visión completa de la realidad de todos los indicadores y variables inherentes al agua [15].

En ese contexto, y después de reconocer las limitaciones de indicadores referentes a la medición de la situación del agua, Mlote, Sullivan y Meigh [16] desarrollan un índice para medir el grado de $\mathrm{PH}$, al que denominan 'Water Poverty Index' (Índice de Pobreza Hídrica, IPH), el cual definen como "una herramienta de medición para evaluar la pobreza en relación con el agua", y establecen como dimensiones de la PH las siguientes: 1. El acceso al agua para consumo humano, 2. El uso que se le da al agua, 3. La capacidad económica y social de las personas para procurarse el agua, 4 . Los recursos hídricos del país o región, y 5. El medio ambiente y su nivel de conservación.
Como se puede observar en estos criterios, los autores están ya proponiendo acciones concretas a tomar sobre las dimensiones de la PH. En tanto que Lawrence et al. [15], construyen un Índice internacional de la PH, e informan sobre la primera fase de un proyecto de investigación para construir una versión local del índice. Este índice les permitió clasificar a 147 países, teniendo en cuenta tanto factores físicos como socioeconómicos, asociados con la escasez de agua en una escala de 0 a 100, donde los valores altos representan menor pobreza hídrica. Cabe mencionar que en este trabajo el IPH para México se estimó en 57.5.

En la evolución de las revisiones al IPH por diversos investigadores, las mejoras van en el sentido de lograr una ponderación más objetiva y analizar la correlación entre las variables e indicadores del IPH mediante la aplicación del análisis de componentes principales (PCA). Cho, Ogwang, y Opio [17] proponen un IPH simplificado de tres indicadores a partir del análisis de la muestra de 147 países análoga a la utilizada en Lawrence et al. [15]. Pérez-Foguet y Giné-Garriga [18] consideran variables como el proceso de captación de agua, las presiones sociales, y las políticas de su manejo, que dan cuenta de la transversalidad de la naturaleza de los problemas del agua y sus impactos. Presentan un WPI mejorado con la técnica de PCA y dirigido a la evaluación de cuencas, y probado en la región de Jequetepeque, Perú. De 25 variables eliminaron 8 , sin eliminar indicadores, pero ajustando sus ponderaciones correspondientes. Otra revisión del IPH, por Jemmali y Sullivan [19], es denominado MWPI, probado en la región de Oriente Medio y Norte de África, contrastando el resultado de eliminar los indicadores Uso y Ambiente con el resultado del IPH original, observando leves diferencias. Para los autores se justifica conservar la integridad del IPH original, ya que la simplificación de un IPH "no debería ir en detrimento de la importante evaluación holística lograda mediante el uso de una estructura convencional del índice".

\section{METODOLOGÍA Y DATOS}

Esta sección se proporciona una explicación breve del IPH y sus ecuaciones. Enseguida se detallan las fases y procesos de cálculo del IPH de este proyecto de investigación.

\section{A. El Índice de Pobreza Hídrica}

Para cumplir los objetivos de la presente investigación, se revisaron diversos métodos de evaluación y medición de la situación del agua, entre ellos la tasa crítica de extracción [20], medición del estrés hídrico [21]; estrés geoespacial por regiones [22]. Se elige el IPH de Lawrence, et al. [15], considerando en su aplicación las revisiones efectuadas al método por Jemmali y Sullivan [19] entre otros.

El IPH es un índice compuesto que consta de 5 indicadores, a saber: Recursos (R), Uso (U), Acceso (A), Capacidad (C), y Medio Ambiente (E). Enseguida se describe brevemente el significado de cada uno de sus dimensiones: a) Recursos (R) se define como la disponibilidad física del agua superficial y subterránea, tomando en cuenta su uso y balance 
hídrico, b) Acceso al agua (A), se define como el nivel de acercamiento al agua segura para uso humano, c) Capacidad de manejo del agua $(\mathrm{C})$, se refiere a la eficacia de la población en el manejo del agua, d) Uso (U), se define como las formas en las cuales el agua es usada para diferentes propósitos por la población, y e) Ambiente (E) este factor trata de captar una serie de indicadores ambientales que reflejan el suministro y la gestión del agua.

A continuación, se anotan y describen las ecuaciones para el cálculo del IPH. La ecuación general para el cálculo del IPH es la (1).

$$
I P H=\frac{\sum_{i=1}^{N} W_{i} X_{i}}{\sum_{i=1}^{N} W_{i}}
$$

Donde: IPH, es el índice de pobreza del agua para una región en particular, siendo el resultado de la suma ponderada de $i$ componentes que se involucran en su cálculo. Los componentes se ponderan con respecto a su importancia relativa, usando funciones de peso $W i$, el peso $W$ se aplica a cada componente $(X i)$ de la estructura del IPH para la región, $X i$ es el valor de cada componente. La ecuación (2) muestra el desarrollo En el IPH propuesto por Lawrence et al. [15], que consta de cinco componentes.

$$
I P H=\frac{W_{r} R+W_{a} A+W_{c} C+W_{u} U+W_{e} E}{W_{r}+W_{a}+W_{c}+W_{u}+W_{e}}
$$

\section{RESULTADOS}

A continuación, se describe el proceso y los resultados obtenidos en la prueba del IPH para México,

\section{A. Fases y procesos del cálculo del IPH}

Se detallan los procesos llevados a cabo en cada una de las fases, tal y como lo recomienda la guía de proyectos PMBOK [35]..

\section{1) Fase 1: Definición de la estrategia de cálculo}

Esta fase se lleva a cabo mediante la ejecución de los siguientes procesos.

Proceso 1) Escala de cálculo. La escala elegida es a nivel macro, dado que uno de los objetivos de esta prueba es calcular el IPH para México a nivel país, y establecer una comparación con los países de la OCDE seleccionados para el estudio.

Proceso 2) Respecto a la muestra: es a conveniencia, no probabilística, los sujetos de estudio están integrados por 17 países de la OCDE, entre ellos México, los cuáles se listan a continuación: Australia, Alemania, Bélgica, Canadá, Dinamarca, Eslovaquia, Eslovenia, España, Francia, Grecia, Islandia, Japón, México, Países Bajos, Polonia, Reino Unido y República Checa.

Proceso 3) En este proceso se procede a la búsqueda y localización de los datos para cada una de las variables seleccionadas, las cuáles se listan la Tabla I. Enseguida se describen las fuentes de datos para las variables de los indicadores del IPH.

Recursos (R). Agrega cinco variables, recopiladas de la base de datos AQUASTAT [4]. R1 es una medida de la disponibilidad total de recursos hídricos renovables reales por habitante y año ( $\left.\mathrm{m}^{3} / \mathrm{hab} / \mathrm{año}\right)$. R2 es la tasa de dependencia del agua exterior, expresa el porcentaje de recursos hídricos renovables totales que tienen su origen fuera del país. R3 mide la precipitación media anual en profundidad ( $\mathrm{mm} / \mathrm{año}$ ), como el promedio a largo plazo (en tiempo y espacio) de la profundidad de las precipitaciones endógenas anuales (producidas en el país). R4 es la variabilidad inter-anual, un indicador normalizado de la variación en el suministro de agua entre años, en valores de 0 a 5 . R5 es la variabilidad estacional, un indicador normalizado de la variación en el suministro de agua entre los meses del año, y va de 0 a 5 .

TABLA I.

INDICADORES Y VARIABLES PARA EL CÁLCULO DEL IPH.

\begin{tabular}{|c|c|c|}
\hline Indicador & $\begin{array}{l}\text { Variabl } \\
\text { e }\end{array}$ & Descripción \\
\hline \multirow[t]{5}{*}{ Recursos } & R1: & Disponibilidad de agua \\
\hline & R2: & Tasa de dependencia del agua exterior \\
\hline & R3: & Precipitación pluvial de largo plazo \\
\hline & R4: & Variabilidad inter-anual \\
\hline & R5: & Variabilidad estacional \\
\hline \multirow[t]{3}{*}{ Acceso } & A1: & Acceso a agua limpia total \\
\hline & A2: & Acceso a saneamiento total \\
\hline & A3: & Acceso a irrigación \\
\hline \multirow[t]{3}{*}{ Capacidad } & C1: & Índice de Desarrollo Humano HDI \\
\hline & C2: & Clasificación de la economía Banco Mundial \\
\hline & C3: & Crecimiento económico promedio \\
\hline \multirow[t]{3}{*}{ Uso } & U1: & Consumo doméstico de agua \\
\hline & $\mathrm{U} 2$ : & Eficiencia de uso de agua en agricultura \\
\hline & U3: & Eficiencia de uso de agua en industria \\
\hline \multirow[t]{3}{*}{ Ambiente } & E1: & Índice de calidad del agua \\
\hline & E2: & Consumo de fertilizante \\
\hline & E3: & Porcentaje de área forestal \\
\hline
\end{tabular}
Fuente: elaboración propia, en base a Lawrence et al. [15] y Jemmali [23].

Acceso (A). Formado a partir de tres variables, las dos primeras tomadas de la base de datos de Progresos en materia de agua potable, saneamiento e higiene [24]. A1 es el acceso a agua segura, medido como el porcentaje de la población con acceso a fuentes de agua mejoradas y con gestión segura. A2 es el acceso a saneamiento total medido como el porcentaje de la población con acceso a servicio de saneamiento gestionado de manera segura. A3 acceso a irrigación, representado por el porcentaje de la superficie con gestión del agua para uso agrícola equipada para el riego, con datos consultados en AQUASTAT [4].

Capacidad (C). Este indicador agrega tres indicadores socioeconómicos consultados de varias fuentes. $\mathrm{C} 1$ es el Índice de Desarrollo Humano (HDI) publicado en el Informe sobre Desarrollo Humano [25]. Este índice compuesto integra a su vez tres dimensiones básicas: la capacidad de llevar una vida larga y saludable, la capacidad de adquirir conocimientos, y la capacidad de lograr un nivel de vida decente. Se miden 
respectivamente mediante la esperanza de vida al nacer, años promedio y esperados de escolaridad, y el ingreso nacional bruto per cápita. C2 es la clasificación de la economía por el Banco Mundial, consultado de sus tablas de indicadores [36] expresada en 4 clases: altos ingresos, ingresos medios altos, ingresos medios bajos, e ingresos bajos. $\mathrm{C} 3$ es calculado como el promedio de los crecimientos anuales del PIB per cápita en los años 2006 a 2015, con datos publicados por AQUASTAT [4].

TABLA II.

DATOS DE LAS VARIABLES DE LOS INDICADORES DEL IPH. Fuente: Elaboración propia, con datos de AQUASTAT [4], OMS/UNICEF [24], World Bank [36].

\begin{tabular}{|c|c|c|c|c|c|c|c|c|c|c|c|c|c|c|c|c|c|c|}
\hline PAÍS & $\mathrm{R} 1$ & $\log R 1$ & $\mathrm{R} 2$ & $\mathrm{R} 3$ & $\mathrm{R} 4$ & $\mathrm{R} 5$ & A1 & $\mathrm{A} 2$ & $\mathrm{~A} 3$ & $\mathrm{Cl}$ & $\mathrm{C} 2$ & $\mathrm{C} 3$ & U1 & $\mathrm{U} 2$ & U3 & E1 & $\mathrm{E} 2$ & $\mathrm{E} 3$ \\
\hline Australia & 20527 & 4.31 & 0 & 534 & 3.2 & 2 & 98 & 74 & 100 & 0.94 & 100 & 1.11 & 26.77 & 0.12 & 4.35 & 85.17 & 53.18 & 16.24 \\
\hline Alemania & 1909 & 3.28 & 30.52 & 700 & 1.1 & 0.8 & 99 & 95 & 100 & 0.93 & 100 & 1.48 & 19.95 & 0.48 & 1.26 & 85.59 & 198.78 & 32.73 \\
\hline Bélgica & 1620 & 3.21 & 30.43 & 847 & 1 & 1.4 & 98 & 97 & 100 & 0.90 & 100 & 0.54 & 15.19 & 0.83 & 0.66 & 75.7 & 276.41 & 22.57 \\
\hline Canadá & 80746 & 4.91 & 1.792 & 537 & 1.2 & 1.6 & 98 & 77 & 100 & 0.92 & 100 & 0.58 & 14.04 & 0.26 & 1.13 & 92.52 & 105.18 & 38.17 \\
\hline Dinamarca & 1058 & 3.02 & 0 & 703 & 1 & 1.3 & 97 & 93 & 100 & 0.93 & 100 & 0.22 & 50.05 & 0.03 & 0.70 & 81.52 & 132.21 & 14.58 \\
\hline Eslovaquia & 9233 & 3.97 & 74.85 & 824 & 0.9 & 0.6 & 93 & 82 & 100 & 0.85 & 100 & 3.63 & 46.31 & 0.99 & 0.48 & 70.74 & 108.88 & 40.35 \\
\hline Eslovenia & 15411 & 4.19 & 41.42 & 1162 & 0.9 & 1 & 98 & 76 & 100 & 0.89 & 100 & 0.75 & 18.38 & 4.86 & 3.77 & 97.62 & 207.51 & 61.97 \\
\hline España & 2418 & 3.38 & 0.269 & 636 & 2.3 & 2.1 & 98 & 97 & 100 & 0.88 & 100 & -0.06 & 14.34 & 0.04 & 8.68 & 81.83 & 109.01 & 36.82 \\
\hline Francia & 3277 & 3.52 & 5.213 & 867 & 1.2 & 1.5 & 93 & 92 & 100 & 0.90 & 100 & 0.35 & 18.99 & 0.17 & 1.82 & 77.44 & 160.18 & 31.03 \\
\hline Grecia & 6244 & 3.80 & 15.2 & 652 & 1.7 & 2.5 & 99 & 75 & 100 & 0.87 & 100 & -1.97 & 14.29 & 0.05 & 6.04 & 86.62 & 80.48 & 31.45 \\
\hline Islandia & 516090 & 5.71 & 0 & 1940 & 1 & 0.8 & 98 & 69 & 0 & 0.92 & 100 & 0.76 & 43.94 & 0.13 & 8.61 & 57 & 147.75 & 0.49 \\
\hline Japón & 3397 & 3.53 & 0 & 1668 & 1.1 & 1.7 & 97 & 100 & 100 & 0.90 & 100 & 0.59 & 18.84 & 0.02 & 1.50 & 87.2 & 208.16 & 68.46 \\
\hline México & 3637 & 3.56 & 11.53 & 758 & 2.5 & 3.1 & 43 & 45 & 100 & 0.76 & 66 & 0.74 & 14.65 & 0.05 & 3.79 & 70.97 & 91.45 & 33.97 \\
\hline Países Bajos & 5377 & 3.73 & 87.91 & 778 & 1 & 0.9 & 100 & 97 & 100 & 0.92 & 100 & 0.72 & 11.41 & 1.65 & 0.40 & 78.49 & 248.99 & 11.16 \\
\hline Polonia & 1567 & 3.20 & 11.4 & 600 & 1.2 & 0.6 & 94 & 77 & 100 & 0.86 & 100 & 3.93 & 17.71 & 0.29 & 4.14 & 80.81 & 169.42 & 30.81 \\
\hline Reino Unido & 2271 & 3.36 & 1.361 & 1220 & 1 & 1.7 & 96 & 98 & 100 & 0.91 & 100 & 0.41 & 74.6 & 0.05 & 0.84 & 90.48 & 244.88 & 33.9 \\
\hline Rep Checa & 1247 & 3.10 & 0 & 677 & 1.3 & 0.6 & 98 & 82 & 100 & 0.88 & 100 & 1.79 & 37.52 & 0.89 & 1.74 & 41.89 & 149.08 & 34.54 \\
\hline
\end{tabular}

Uso (U). Integrado por tres variables, con datos provenientes de FAOSTAT [26]. U1 presenta la extracción de agua municipal como porcentaje de la extracción total. U2 es un cálculo de la eficiencia de uso de agua en agricultura, como el cociente de dos datos reportados: extracción de agua agrícola como porcentaje de extracción total y valor agregado de la agricultura como porcentaje del PIB. De modo similar, U3 es un cálculo de la eficiencia de uso de agua en la industria, calculado como el cociente de dos datos reportados: extracción de agua para uso industrial como porcentaje de extracción total y valor agregado de la industria como porcentaje del PIB.

Ambiente (E). Agrega tres variables. E1 es un índice de calidad del agua tomado de una estimación internacional que combina un Índice de Desempeño Ambiental (Environmental Performance Index) con un Índice de Calidad de Agua (Water Quality Index) [27], en base a cinco parámetros de calidad comúnmente reportados: oxígeno disuelto, conductividad eléctrica, valor del $\mathrm{pH}$, y concentraciones de nitrógeno y fósforo. E2 es una medida del consumo de fertilizante por área cultivada, en $\mathrm{kg} / \mathrm{ha}$. E3 es el porcentaje del área forestal

respecto al área total del territorio del país; E2 y E3 se obtienen de FAOSTAT [26].

Los datos recopilados en este proceso se presentan en la Tabla II.

2) Fase 2: Tratamiento estadístico de los datos previo al cálculo del IPH

Proceso 1) Al considerar los valores extremos, se procede a la conversión a escala logarítmica, Eq. (3), de la variable R1 del componente Recursos (R), ya que como se puede observar en la Tabla II, los datos de origen presentan valores en un rango demasiado amplio entre 1050 (Dinamarca) y 516,090 (Islandia). Con esto se previene una distorsión en el indicador Recursos. Se muestran los resultados en la columna "log R1" de la Tabla II. Respecto al resto de los datos, desde la fuente de origen se presentan en una escala comparable, por lo que no se les aplica la conversión del logaritmo.

Proceso 2) Normalización de los datos por el método MinMax, de tal manera que cada serie de datos quede con una puntuación entre 0 y 100 , donde 0 representa el valor que conlleva a una mayor PH, y 100 el que denota una menor PH. Para normalizar los datos se utiliza la ecuación (4).

Proceso 3) Normalización de los datos por el método MinMax, para el caso de variables cuyo sentido es contrario a la escala del IPH, para ello se utiliza la Ec. (5). Los resultados se muestran en la Tabla III.

$$
\begin{aligned}
x^{*} & =\log (x) \\
x_{i}^{*} & =100 \frac{X_{\max }-X_{i}}{X_{\max }-X_{\min }} \\
x_{i}^{*} & =100 \frac{X_{i}-X_{\min }}{X_{\max }-X_{\min }}
\end{aligned}
$$

Proceso 4) Conversión de valores cualitativos a rangos de porcentajes de 0 a 100 . En este estudio el dato de la variable C2 está expresada de origen por el Banco Mundial en 4 clases (altos ingresos, ingresos medios, ingresos medios bajos, e ingresos bajos). Los resultados se presentan en la Tabla III, columna $\mathrm{C} 2$.

\section{3) Fase 3: Cálculo de los indicadores para cada} dimensión del IPH.

Los resultados para toda la muestra se presentan en la Tabla IV, y además para México los indicadores se presentan en la Fig. 1. En la Tabla IV se observa que México tiene el menor valor en los indicadores R, A y C. Por otro lado, en el indicador U ocupa el lugar 6, con un valor de 45.5 y en el indicador E está en el lugar 8. Los países que ocupan el primer lugar por cada dimensión son Islandia en R, Japón en A, Polonia en C, Eslovenia en U y Canadá en E. 
4) Fase 4: Cálculo del IPH por el método tradicional

Con los promedios de las variables de los indicadores del IPH de la Tabla IV se procede a calcular el IPH, para ello se utilizan las ecuaciones 1 y 2 . De acuerdo con el método se selecciona el criterio de ponderaciones iguales $W_{r}=W_{a}=$ $W_{c}=W_{u}=W_{e}=0.20$. Los resultados se muestran en la Tabla IV en la columna IPH, y en la Fig. 2. La mayor PH de la muestra la presenta México, con un IPH de 41.6 y los indicadores que tienen la mayor contribución a su $\mathrm{PH}$ son Recursos, Acceso y Capacidad, con valores de 30.6, 33.6, y 37.3.

5) Fase 5: Pruebas de balance estadístico entre las variables de los indicadores, y entre el IPH y los indicadores (o pruebas factibilidad de PCA).

Esta fase realiza pruebas estadísticas para observar la correlación entre variables y así estar en condiciones de descartar aquellas que contribuyen con un doble conteo; entre las técnicas más citadas en la literatura se encuentran la de PCA y el análisis de correlación de orden [28], [29], [30].

Proceso 1) Prueba de Correlación de Pearson, se consideran los siguientes criterios: correlación muy alta $r>0.7$, alta $0.6<r<0.7$, moderada $0.5<r<0.6$ y baja $r<0.5$. Los resultados se muestran en la Tabla $\mathrm{V}$; en el cual se puede observar que existe correlación entre las variables R4 y R5, A2 y A1, C1y C2, y no existe correlación entre las variables de U y E.

TABLA III.

RESUltAdOS DE LA NORMALIZACIÓN DE VARIABLES POR EL MÉTODO MIN - MAX .

\begin{tabular}{|c|c|c|c|c|c|c|c|c|c|c|c|c|c|c|c|c|c|}
\hline PAÍS & $\mathrm{R} 1$ & $\mathrm{R} 2$ & $\mathrm{R} 3$ & $\mathrm{R} 4$ & $\mathrm{R} 5$ & $\mathrm{~A} 1$ & $\mathrm{~A} 2$ & $\mathrm{~A} 3$ & $\mathrm{C} 1$ & $\mathrm{C} 2$ & $\mathrm{C} 3$ & $\mathrm{U} 1$ & $\mathrm{U} 2$ & U3 & $\mathrm{E} 1$ & $\mathrm{E} 2$ & E3 \\
\hline Australia & 47.91 & 100.00 & 0.00 & 0.00 & 44.00 & 96.49 & 52.73 & 100.00 & 100.00 & 100.00 & 52.28 & 75.69 & 2.18 & 47.67 & 77.66 & 100.00 & 25.17 \\
\hline Alemania & 53 & 65.28 & 11.81 & 91.30 & 92.00 & 98.25 & 90.91 & 100.00 & 66 & 100.00 & 58.46 & 8 & 9.62 & 10.38 & 78.41 & 4.78 & 17.43 \\
\hline Bélgica & 6.88 & 65.39 & 22.26 & 95.65 & 68.00 & 96.49 & 94.55 & 100.00 & 75.71 & 100.00 & 42.53 & 94.02 & 16.77 & 3.12 & 60.67 & 0.00 & 32.48 \\
\hline Canadá & 70.03 & 97.96 & 0.21 & 86.96 & 60.00 & 96.49 & 58.18 & 100.00 & 89.27 & 100.00 & 43.31 & 95.84 & 5.00 & 8.82 & 90.85 & 76.71 & 55.44 \\
\hline Dinamarca & 0.00 & 100.00 & 12.02 & 95.65 & 72.00 & 94.74 & 87.27 & 100.00 & 92.09 & 100.00 & 37.12 & 38.85 & 0.33 & 3.64 & 71.11 & 64.60 & 20.73 \\
\hline Eslovaquia & 35.00 & 14.86 & 20.63 & 100.00 & 100.00 & 87.72 & 67.27 & 100.00 & 46.89 & 100.00 & 94.93 & 44.77 & 20.13 & 0.97 & 51.77 & 75.05 & 58.64 \\
\hline Eslovenia & 43.28 & 52.88 & 44.67 & 100.00 & 84.00 & 96.49 & 56.36 & 100.00 & 72.32 & 100.00 & 46.21 & 88.97 & 100.00 & 40.66 & 100.00 & 30.87 & 90.45 \\
\hline España & 13.35 & 99.69 & 7.25 & 39.13 & 40.00 & 96.49 & 94.55 & 100.00 & 68.93 & 100.00 & 32.47 & 95.36 & 0.43 & 100.00 & 71.67 & 74.99 & 53.45 \\
\hline Francia & 18.26 & 94.07 & 23.68 & 86.96 & 64.00 & 87.72 & 85.45 & 100.00 & 76.27 & 100.00 & 39.38 & 88.00 & 3.22 & 17.18 & 63.79 & 52.07 & 44.93 \\
\hline Grecia & 28.68 & 82.71 & 8.39 & 65.22 & 24.00 & 98.25 & 54.55 & 100.00 & 58.76 & 100.00 & 0.00 & 95.44 & 0.64 & 68.14 & 80.26 & 87.77 & 45.55 \\
\hline Islandia & 100.00 & 100.00 & 100.00 & 95.65 & 92.00 & 96.49 & 43.64 & 0.00 & 89.83 & 100.00 & 46.31 & 48.52 & 2.26 & 99.12 & 27.11 & 57.64 & 0.00 \\
\hline Japón & 18.85 & 100.00 & 80.65 & 91.30 & 56.00 & 94.74 & 100.00 & 100.00 & 79.66 & 100.00 & 43.38 & 88.24 & 0.00 & 13.27 & 81.30 & 30.57 & 100.00 \\
\hline México & 19.95 & 86.88 & 15.93 & 30.43 & 0.00 & 0.00 & 0.00 & 100.00 & 0.00 & 66.00 & 46.03 & 94.87 & 0.65 & 40.92 & 52.18 & 82.86 & 49.26 \\
\hline Países Bajos & 26.26 & 0.00 & 17.35 & 95.65 & 88.00 & 100.00 & 94.55 & 100.00 & 91.53 & 100.00 & 45.61 & 100.00 & 33.78 & 0.00 & 65.67 & 12.28 & 15.70 \\
\hline Polonia & 6.35 & 87.03 & 4.69 & 86.96 & 100.00 & 89.47 & 58.18 & 100.00 & 52.54 & 100.00 & 100.00 & 90.03 & 5.61 & 45.12 & 69.84 & 47.93 & 44.61 \\
\hline Reino Unido & 12.34 & 98.45 & 48.79 & 95.65 & 56.00 & 92.98 & 96.36 & 100.00 & 83.05 & 100.00 & 40.31 & 0.00 & 0.67 & 5.32 & 87.19 & 14.12 & 49.15 \\
\hline Rep Checa & 2.66 & 100.00 & 10.17 & 82.61 & 100.00 & 96.49 & 67.27 & 100.00 & 65.54 & 100.00 & 63.83 & 58.68 & 18.10 & 16.19 & 0.00 & 57.04 & 50.10 \\
\hline
\end{tabular}

TABLA IV.

INDICADORES R, A, C, U, E E IPH CALCULADOS.

\begin{tabular}{|r|r|r|r|r|r|r|}
\hline PAIS & $\mathrm{R}$ & $\mathrm{A}$ & $\mathrm{C}$ & $\mathrm{U}$ & $\mathrm{E}$ & $\mathrm{IPH}$ \\
\hline Australia & 38.4 & 83.1 & 84.1 & 41.8 & 66.9 & 62.9 \\
\hline Alemania & 54.0 & 96.4 & 83.7 & 35.5 & 53.5 & 64.6 \\
\hline Bélgica & 51.6 & 97.0 & 72.7 & 38.0 & 31.1 & 58.1 \\
\hline Canadá & 63.0 & 84.9 & 77.5 & 36.6 & 74.3 & 67.3 \\
\hline Dinamarca & 55.9 & 94.0 & 76.4 & 14.3 & 52.1 & 58.6 \\
\hline Eslovaquia & 54.1 & 85.0 & 80.6 & 22.0 & 61.8 & 60.7 \\
\hline Eslovenia & 65.0 & 84.3 & 72.8 & 76.5 & 73.8 & 74.5 \\
\hline España & 39.9 & 97.0 & 67.1 & 65.3 & 66.7 & 67.2 \\
\hline Francia & 57.4 & 91.1 & 71.9 & 36.1 & 53.6 & 62.0 \\
\hline Grecia & 41.8 & 84.3 & 52.9 & 54.7 & 71.2 & 61.0 \\
\hline Islandia & 97.5 & 46.7 & 78.7 & 50.0 & 28.2 & 60.2 \\
\hline Japón & 69.4 & 98.2 & 74.3 & 33.8 & 70.6 & 69.3 \\
\hline México & 30.6 & 33.3 & 37.3 & 45.5 & 61.4 & 41.6 \\
\hline Paises Bajos & 45.5 & 98.2 & 79.0 & 44.6 & 31.2 & 59.7 \\
\hline Polonia & 57.0 & 82.6 & 84.2 & 46.9 & 54.1 & 65.0 \\
\hline Reino Unido & 62.2 & 96.4 & 74.5 & 2.0 & 50.2 & 57.1 \\
\hline Rep Checa & 59.1 & 87.9 & 76.5 & 31.0 & 35.7 & 58.0 \\
\hline
\end{tabular}

Proceso 2) Prueba de esfericidad de Bartlett entre las variables de los indicadores, para probar la hipótesis nula de que los indicadores individuales en una matriz de correlación no están correlacionados [31], [23]. Los resultados se muestran en la Tabla VI, columnas R, A, C, U, y E.

Proceso 3) Prueba de medida de adecuación del muestreo de Kaiser Meyer Olkin (KMO) sirve para comparar las magnitudes de los coeficientes de correlación observados con las de los coeficientes de correlación parcial [32]. En esta prueba se considera que la muestra es viable para PCA con valores mayores a 0.5 , los resultados se presentan en la Tabla VI, columnas R, A, C, U, y E.

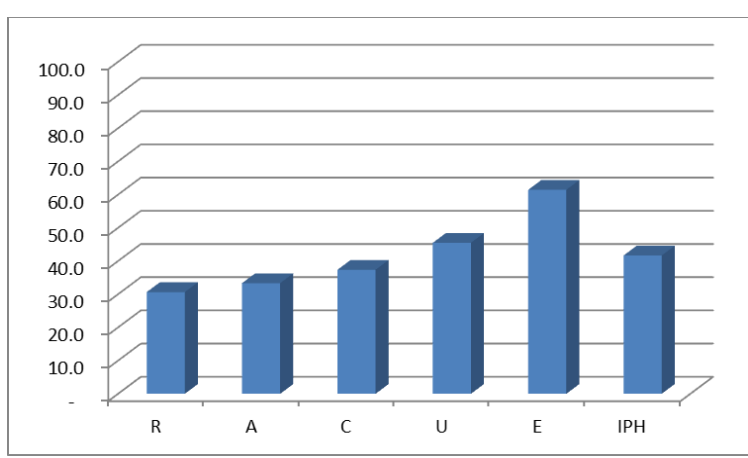

Fig 1. Indicadores e Índice de Pobreza Hídrica estimados para México.

Como se puede observar en la Tabla VI, las pruebas de esfericidad de Bartlett de las variables R, A, y C, indican que PCA es viable, sin embargo, la prueba KMO, de acuerdo con los criterios establecidos indica que PCA no es viable, lo que significa que no se descartan variables y el IPH se calcula con la selección de la Tabla I. 
TABLA V.

COEFICIENTES DE CORRELACIÓN DE LAS VARIABLES DE LOS INDICADORES R, A, C, U, Y E.

\begin{tabular}{|c|c|c|c|c|c|c|c|c|c|}
\hline & R1 & R2 & R3 & R4 & R5 & & & \multirow{3}{*}{ A2 } & \multirow{3}{*}{ A3 } \\
\hline R1 & & 10.02 & $20 \quad 0.431$ & -0.033 & 0.045 & \multirow{2}{*}{\multicolumn{2}{|c|}{ A1 }} & & \\
\hline R2 & 0.020 & 201.00 & $00 \quad 0.111$ & -0.342 & -0.378 & & & & \\
\hline R3 & 0.431 & $\begin{array}{ll}31 & 0.11 \\
\end{array}$ & $11 \quad 1.000$ & 0.390 & 0.157 & A1 & 1.000 & 0.689 & -0.079 \\
\hline R4 & -0.033 & $33-0.34$ & $\begin{array}{ll}42 & 0.390\end{array}$ & 1.000 & 0.680 & A2 & 0.689 & $\begin{array}{l}9.000 \\
\end{array}$ & 0.266 \\
\hline \multirow[t]{3}{*}{ R5 } & 0.045 & $\begin{array}{ll}15 & -0.37 \\
\end{array}$ & $\begin{array}{ll}78 & 0.157 \\
\end{array}$ & 0.680 & 1.000 & A3 & -0.079 & $\begin{array}{l}9 \\
9\end{array}$ & 1.000 \\
\hline & 0.18 & $\begin{array}{ll}39 & 0.27\end{array}$ & $\begin{array}{ll}73 & 0.374\end{array}$ & 0.733 & 0.632 & & 0.481 & 0.546 & 0.077 \\
\hline & $\mathrm{C} 1$ & $\mathrm{C} 2$ & $\mathrm{C} 3$ & & & U1 & U2 & U3 & \\
\hline C1 & 1.000 & $\begin{array}{ll}0 & 0.77\end{array}$ & $79-0.195$ & & U1 & 1.000 & 0.166 & 0.176 & \\
\hline $\mathrm{C} 2$ & 0.779 & $\begin{array}{ll}9 & 1.00 \\
9\end{array}$ & $00 \quad 0.033$ & & U2 & 0.166 & 1.000 & -0.109 & \\
\hline \multirow[t]{3}{*}{ C3 } & -0.195 & $\begin{array}{ll}5 & 0.03 \\
\end{array}$ & $\begin{array}{ll}33 & 1.000 \\
\end{array}$ & & U3 & 0.176 & -0.109 & 1.000 & \\
\hline & 0.645 & $\begin{array}{ll}5 & 0.60 \\
5\end{array}$ & $\begin{array}{ll}08 \quad 0.039 \\
\end{array}$ & & & 0.059 & 0.039 & 0.043 & \\
\hline & E1 & E2 & E3 & & & & & & \\
\hline E1 & 1.000 & -0.110 & 0.388 & & & & & & \\
\hline E2 & -0.110 & 1.000 & -0.106 & & & & & & \\
\hline \multirow[t]{2}{*}{ E3 } & 0.388 & -0.106 & 1.000 & & & & & & \\
\hline & 0.1624 & 0.0233 & 0.1616 & & & & & & \\
\hline
\end{tabular}

\section{IPH}

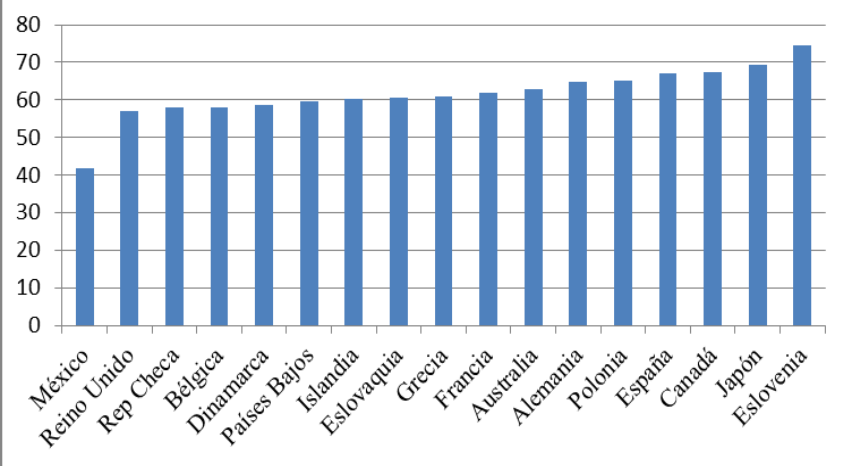

Fig. 2. Índices de Pobreza Hídrica estimados para la muestra de países.

\section{6) Fase 6: Pruebas de balance estadístico entre los indicadores del IPH,}

Proceso 1) Correlación de Pearson, los resultados se muestran en la Tabla VII, como se puede observar la correlación entre los indicadores del IPH es moderada entre A y C, y para el resto de los indicadores la correlación es baja, de acuerdo con los criterios establecidos. Así mismo en la Tabla VIII se exponen las correlaciones de Pearson del IPH con cada indicador las cuales son R: 0.358, A: 0.557, C: 0.573 , U: 0.368 , y E: 0.386 .

Proceso 2) Correlación de Kendall (de orden) [33], que mide el grado de correspondencia entre dos clasificaciones y el significado estadístico de esta asociación. Los resultados se muestran en la Tabla VII con correlaciones aún más bajas que con la prueba estadística de Pearson.

Proceso 3) Esfericidad de Bartlett de significancia de existencia de correlación para los indicadores. Los resultados presentan una significancia de 0.170 , como se muestran en la Tabla VI columna IPH, y de acuerdo el criterio establecido no es viable realizar PCA para descartar indicadores.

Proceso 4) Prueba KMO, los resultados se muestran en la
Tabla VI, columna IPH, presentan un coeficiente KMO de 0.45 , y de acuerdo con el criterio establecido, no existe viabilidad de aplicar PCA.

TABLA VI.

PRUEBA DE ESFERICIDAD DE BARTLETT Y PRUEBA KMO ENTRE LAS VARIABLES DE LOS INDICADORES R, A, C, U, Y E, Y ENTRE INDICADORES DEL IPH.

\begin{tabular}{|c|c|c|c|c|c|c|}
\hline Pruebas estadísticas & $\mathbf{R}$ & A & C & U & $\mathbf{E}$ & IPH \\
\hline \multicolumn{7}{|l|}{ Esfericidad de Bartlett } \\
\hline n observaciones & 17 & 17 & 17 & 17 & 17 & 17 \\
\hline k variables & 5 & 3 & 3 & 3 & 3 & 5 \\
\hline$n / k$ ( $<5$ recomendable) & 3.40 & 5.67 & 5.67 & 5.67 & 5.67 & 3.40 \\
\hline det mat corr & 0.25 & 0.42 & 0.34 & 0.92 & 0.84 & 0.35 \\
\hline estadistico B & 18.48 & 12.31 & 15.14 & 1.13 & 2.55 & 14.06 \\
\hline grados lib & 10.00 & 3.00 & 3.00 & 3.00 & 3.00 & 10.00 \\
\hline valor-p (<0.05 PCA ok) & 0.047 & 0.006 & 0.002 & 0.769 & 0.467 & 0.170 \\
\hline PCA viable & $\mathrm{SI}$ & $\mathrm{SI}$ & $\mathrm{SI}$ & NO & NO & NO \\
\hline \multicolumn{7}{|l|}{ Kaiser Meyer Olkin } \\
\hline indice (>0.50 PCA ok) & 0.46 & 0.38 & 0.43 & 0.42 & 0.53 & 0.45 \\
\hline PCA viable & NO & NO & NO & NO & $\mathrm{SI}$ & NO \\
\hline
\end{tabular}

TABLA VII.

MATRICES DE CORRELACión DE PEARSON Y DE KENDALl ENTE LOS INDICADORES DEL IPH.

\begin{tabular}{|c|c|c|c|c|c|c|c|c|c|c|c|}
\hline (Pearson) & $R$ & A & C & $\mathrm{U}$ & $\mathrm{E}$ & (Kendal) & R & A & C & $\mathrm{U}$ & $\mathrm{E}$ \\
\hline$R$ & 1000 & & & & & R & 1.000 & & & & \\
\hline A & .0090 & 1000 & & & & A & -0022 & 1000 & & & \\
\hline C & 0.457 & 0.524 & 1.000 & & & C & 0.118 & .0 .111 & 1.000 & & \\
\hline U & .0097 & .0243 & .0251 & 1000 & & U & .0266 & .0273 & .0118 & 1000 & \\
\hline $\mathrm{E}$ & .0303 & 0.051 & .0.1248 & 0.251 & 1.000 & $\mathrm{E}$ & .0.059 & .0 .155 & 0.118 & 0.266 & 1.000 \\
\hline
\end{tabular}

TABLA VIII.

CORRELACIÓN DE PEARSON ENTE IPH Y SUS INDICADORES

\begin{tabular}{|l|l|l|l|l|l|}
\hline & $\mathrm{R}$ & $\mathrm{A}$ & $\mathrm{C}$ & $\mathrm{U}$ & $\mathrm{E}$ \\
\hline $\mathrm{nIPH}$ & 0.358 & 0.557 & 0.573 & 0.368 & 0.386 \\
\hline
\end{tabular}

7) Fase 7: Cálculo del IPH con los indicadores resultantes del análisis de PCA.

Esta prueba no se lleva a cabo en este proyecto, derivado de los resultados obtenidos en el análisis estadístico, que se muestra en la Tabla VI y la Tabla VII, se determina la no viabilidad de aplicar PCA derivado de la escasa correlación existente entre las variables, y entre los indicadores.

\section{DISCUSIÓN DE RESULTADOS}

Se procede a analizar los resultados del IPH obtenidos mediante la estrategia de cálculo diseñada para este ejercicio en la cual el IPH se considera que es el que mejor mide la PH pese a sus limitaciones y críticas, pues es el que mejor integra las dimensiones del desarrollo humano y va más allá de las variables físicas de disponibilidad y cobertura.

En la Tabla IV se presentan los resultados que corresponden al cálculo por el método original sin eliminar variables ni indicadores. En este análisis se puede observar 
que la mayor PH la presenta México, con un valor del IPH de 41.6, por lo que se cumple la hipótesis planteada en esta investigación; por otro lado, en la Tabla IV también se observa que la menor PH la presenta Eslovenia con un valor de IPH de 74.5. En este mismo contexto los resultados arrojan que Japón, Canadá, España, Polonia, Alemania, Australia, Francia, Grecia, Eslovaquia e Islandia tienen un IPH arriba de 60.0 y entre los países que presentan un IPH menor que 60.0, al igual que México, se encuentran Países Bajos, Dinamarca, Bélgica, República Checa y Reino Unido.

En el caso de México, los indicadores que presentan una mayor contribución a la $\mathrm{PH}$ son $\mathrm{R}$, A y C, con valores de 30.6, 33.6, y 37.3 (Tabla IV), de lo cual se puede inferir que es resultado de la baja disponibilidad de agua per cápita en el caso de R; y en el caso de A, a pesar de la alta cobertura de agua y saneamiento reportada por las instituciones mexicanas, no califica como agua y saneamiento seguros, según los estándares establecidos en el ODS 6; por lo que estas variables (A1 y A2) fueron castigadas severamente en el reporte de OMS/UNICEF [24] como se muestran en la Tabla II, situación que influyó en el resultado del bajo IPH para México, y respecto al indicador $\mathrm{C}$, éste involucra variables económicas C2 (ingreso) y de desarrollo C1 (IDH), aspectos en los que México tampoco sobresale.

Desde la lectura de la Tabla VIII se observa el grado de correlación entre el IPH y los indicadores en los países de la muestra. La mayor correlación la presenta el indicador $\mathrm{C}$, con 0.573, seguido de A con 0.557, consideradas moderadas de acuerdo con los criterios establecidos en la fase 6 , proceso 1 de la estrategia de cálculo. Los indicadores $\mathrm{R}, \mathrm{U}$ y $\mathrm{E}$ tienen una correlación baja con el IPH. La mayor correlación entre el IPH y C, se explica porque $\mathrm{C}$ involucra las variables socioeconómicas: IDH, clasificación de la economía según ingresos, y crecimiento económico promedio; lo que refuerza el nexo de la pobreza con la capacidad de gestión del agua, tanto por los países como por las personas [34], [16].

Desde el punto de vista de los resultados del balance estadístico de las variables y los indicadores, el hecho de que no resultara viable la aplicación de PCA, se explica en parte porque desde la fase 1, proceso 3 de la metodología, se hizo el esfuerzo de seleccionar solamente las variables cuya información fuera excluyente entre sí. Como ejemplo, se incluyeron solamente A1 y A2 (agua y saneamiento totales) y se dejó fuera el agua y saneamiento rural, variables que fueron eliminadas por medio de PCA en [23].

\section{CONCLUSIONES}

La carencia o escasez en relación con el agua, su uso y distribución eficiente, la disponibilidad y acceso, son un tema que ha venido cobrando fuerza en las sociedades modernas, ante ello, el propósito de esta investigación es indagar por medio del IPH el grado de la PH en México en comparación con la de algunos países de la OCDE; así como implementar una estrategia de cálculo para el IPH, analizar la influencia de los indicadores que más contribuyen a la $\mathrm{PH}$ de México, e indagar el grado de correlación entre el IPH y la pobreza de ingresos en la muestra de países.

En este estudio se diseñó una estrategia de cálculo del IPH y se estimó el grado de PH de México en una comparación con 16 países de la OCDE. En el proceso se halló innecesaria la eliminación de variable alguna y se conservaron los cinco indicadores que componen el IPH: R, A, C, U y E. Se comprobó con un método objetivo la importancia de la selección de variables.

Respecto al resultado de 41.6 del IPH para México, que fue calculado a escala macro, ha de interpretarse con cautela, dada la diversidad de ecosistemas y situaciones socioeconómicas existente en y entre las regiones del país, pues es sabido que los promedios esconden la realidad del agua a escalas locales.

Tal y como se planteó en la hipótesis, México presentó el menor IPH de la muestra de países, esto es, la mayor PH, y los indicadores que contribuyeron notablemente a la $\mathrm{PH}$ de México son $\mathrm{R}$, A y $\mathrm{C}$, con valores que son también los menores entre los países del estudio.

En la selección de países se encontró que el indicador socioeconómico C influye sobre el IPH más que los demás indicadores, aunque en grado moderado.

\section{Recomendaciones}

Las perspectivas futuras para el diseño de la metodología de cálculo del IPH tienen que ver con su vinculación a las políticas públicas del agua, tales como: 1) incluir como primer paso la presentación del proyecto a las entidades encargadas de tomar decisiones relacionadas con el agua, para que el ejercicio trascienda más allá de la academia, 2) calcular el índice a otras escalas que tomen en cuenta las realidades locales, para la focalización de acciones, 3) repetir periódicamente la prueba del IPH para monitorear los cambios en el índice y sus dimensiones, y 4) atender integralmente la problemática del agua, para evitar la incompleta visión que representa la sola medida de cobertura de servicios de agua y saneamiento.

\section{AgRADECIMIENTOS}

Este trabajo comprende parte de los resultados obtenidos en la tesis de doctorado del primer autor, con asesoría del coautor. Se agradece al Consejo Nacional de Ciencia y Tecnología (Conacyt), institución que financió los estudios de doctorado en Desarrollo Regional en el Centro de Alimentación y Desarrollo A.C. (CIAD).

\section{REFERENCIAS}

[1] Sen A. "Capability and Wellbeing". En: The Quality of Life. (M. Nussbaum, A. Sen Ed.). World Institute for Development Economics Research of the United Nations University. Oxford University Press, New York, EUA. pp 30-53. 1993.

[2] Feitelson E. y Chenoweth J. "Water poverty: Towards a meaningful indicator". Water Policy, 4(3), 263-281. 2002. DOI: 10.1016/S13667017(02)00029-6.

[3] ONU. "Resolución 70/1 de la Asamblea General "Transformar nuestro mundo: la Agenda 2030 para el Desarrollo Sostenible" A/RES/70/1, 2015. https://undocs.org/sp/A/RES/70/1 15/03/2019.

[4] FAO. "Base de Datos Principal AQUASTAT". Organización de las Naciones Unidas para la Alimentación y la Agricultura (FAO), 2016a [en http://www.fao.org/nr/water/aquastat/data/query/index.html?lang=es 20/11/2018 
[5] Falkenmark M., Lundqvist J., y Widstrand C. "Macro-scale water scarcity requires micro-scale approaches: Aspects of vulnerability in semi-arid development". Natural resources forum, 13 (4), 258-267. 1989. DOI: $10.1111 /$ j.1477-8947. 1989.tb00348. x.

[6] OECD. "OECD Environmental Outlook to 2050", OECD Publishing. 2012. DOI: $10.1787 / 9789264122246$-en.

[7] CONAGUA. "Estadísticas del agua en México, edición 2018". Comisión Nacional del Agua. Ciudad de México, México. 306 pp. 2018.

[8] OECD. "Better Life Index dataset in OECD Stat". Organisation for Economic Co-Operation and Development. 2019a [en línea] https://stats.oecd.org/Index.aspx? DataSetCode=BLI 30/04/2019.

[9] Townsend P. "Poverty in the United Kingdom: A Survey of Household Resources and Standards of Living". University of California Press, 1216 pp. 1979.

[10] Pigou A. C. "The Economics of Welfare. The Economics of Welfare". 4a ed. Macmillan. London, UK. 550 pp. 1932.

[11] Molle F. y Mollinga P. "Water poverty indicators: Conceptual problems and policy issues". Water Policy, 5(5-6). 529-544. 2003.DOI:10.2166/wp.2003.0034.

[12] Rijsberman F. "Water scarcity: Fact or fiction? Agricultural Water Management". 80 (1-3), 5-22. 2006. DOI: 10.1016/j.agwat.2005.07.001.

[13] Lissner T., Sullivan C., Reusser D. y Kropp J. "Integrated assessments of water scarcity: knowns, unknowns and ways forward". En: The Global Water System in the Anthropocene. (A. Bhaduri, J. Bogardi, J. Leentvaar, S. Marx Eds.). Springer International Publishing, Switzerland. 59-72. 2014 DOI: 10.1007/978-3-319-07548-8.

[14] Marvin S. "Accessibility to utility networks: Local policy issues". Local Government Studies, 20(12). 437-457. 1994. DOI: $10.1080 / 03003939408433738$.

[15] Lawrence P., Meigh J. y Sullivan C. "The Water Poverty Index: an International Comparison". Keele Economics Research Papers. Keele Economics Research, 2002(19), 24 pp. 2003.

[16] Mlote S., Sullivan C. y Meigh J. "Water poverty index: a tool for integrated water management". 3rd WaterNet / Warfsa Symposium "Water Demand Management for Sustainable Development". Dar es Salaam, 30-31 octubre 2002. 2002.

[17] Cho D., Ogwang T., y Opio C. "Simplifying the water poverty index". Social indicators research,97(2). 257-267. 2010.DOI: 10.1007/s11205-009-9501-2.

[18] Giné-Garriga R. y Pérez-Foguet A. "Application of a revised Water Poverty Index to target the water poor". Water Science and technology. 63(6), 1099-1110. 2011. DOI: 10.2166/wst.2011.347.

[19] Pérez-Foguet A. y Gine-Garriga R. "Analyzing Water Poverty in Basins. Water Resource Management”, (7), 3595-3613. 2011.

[20] Jemmali H. y Sullivan C.A. "Multidimensional Analysis of Water Poverty in MENA Region: An Empirical Comparison with Physical Indicators". Social Indicators Research. 115(1), 253-277. 2014. DOI: 10.1007/s11205-012-0218-2.

[21] Raskin P., Gleick P., Kirshen P., Pontius G. y Strzepek K. "Water futures: assessment of long-range patterns and problems". Comprehensive assessment of the freshwater resources of the world. Stockholm Environment Institute. Stockholm, Sweden. 77 pp. 1997.

[22] Seckler D., A. "World water demand and supply, 1990 to 2025 Scenarios and issues" (19). International Water Management Institute.Colombo, Sri Lanka. 40 pp. 1998. DOI: 10.3910/2009.019.

[23] Vörösmarty C.J., Green P., Salisbury J. y Lammers R.B. "Global water resources: vulnerability from climate change and population growth". Science. 289(5477), 284-288. 2000. DOI: 10.1126/science.289.5477.284.

[24] Jemmali H. "Mapping water poverty in Africa using the improved Multidimensional Index of Water Poverty". International journal of water resources development, 33(4), 649-666. 2017.

[25] OMS/UNICEF "Progresos en materia de agua potable, saneamiento e higiene: informe de actualización de 2017 y línea de base de los ODS" [Ginebra: Organización Mundial de la Salud y el Fondo de las Naciones Unidas para la Infancia (UNICEF). 2017 [En línea] https://washdata.org/data/household 21/10/2018.

[26] PNUD "Informe sobre Desarrollo Humano 2016". Programa de las Naciones Unidas para el Desarrollo. 2016 [En línea]
http://hdr.undp.org/sites/default/files/HDR2016_SP_Overview_Web.pdf 23/10/2018.

[27] FAO "Base de Datos FAOSTAT". Organización de las Naciones Unidas para la Alimentación y la Agricultura (FAO), (2016b). [en línea]. http://www.fao.org/faostat/en/\#data 20/11/2018.

[28] Srebotnjak T., Carr G., de Sherbinin A. y Rickwood C. "A global water quality index and hotdeck imputation of missing data". Ecological $\begin{array}{llll}\text { Indicators, } & 17(6), & 108-119 . & 2012 .\end{array}$ https://doi.org/10.1016/j.ecolind.2011.04.023.

[29] Heidecke C. "Development and Evaluation of a Regional Water Poverty Index for Benin", EPTD discussion paper. International Food Policy Research Institute. (145), 38pp. 2006.

[30] Fenwick C. "Identifying the water poor: An indicator approach to assessing water poverty in rural Mexico". Tesis de doctorado Department of civil, environmental and geomatic engineering. University College London. Londres. 270pp. 2010.

[31] Hotelling H. "Analysis of a complex of statistical variables into principal components". Journal of educational psychology, 24(6), 417 441. 1933. DOI: $10.1037 / \mathrm{h} 0071325$.

[32] Nardo M., Saisana, M., Saltelli A., Tarantola S., Hoffman A. y Giovannini E. "Handbook on Constructing Composite Indicators and User Guide". OECD Publishing. Paris, France. 162pp. 2008. DOI: $10.1787 / 533411815016$

[33] Kaiser H.F. y Rice J. "Little Jiffy, Mark IV". Educational and psychological measurement. 34(1). 111-117. 1974 DOI: $10.1177 / 001316447403400115$.

[34] Bonett D. G. y Wright T. A. "Sample size requirements for estimating Pearson, Kendall and Spearman correlations". Psychometrika, 65(1), 2328. 2000. DOI: $10.1007 / \mathrm{BF} 02294183$.

[35] Sen A. "El desarrollo como libertad". Gaceta Ecológica. 2000. [en linea] http://www.redalyc.org/articulo.oa?id=53905501 29/04/2019.

[36] Project Management Institute Inc. "PMBOK Guía de los fundamentos para la dirección de proyectos". Project Management Institute. Pennsylvania. 569 pp. 2013.

[37] World Bank. World International Indicators. Washingto D.C.: World Bank, 2016. DOI:10.1596/978-1-4648-0683-4.

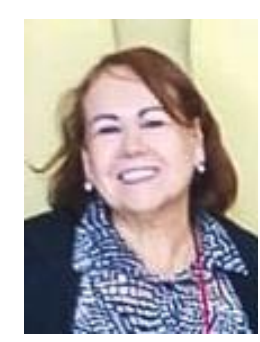

María Juliana Olivas Palma. Candidata a Doctora en Desarrollo Regional (2019), por el Centro de Investigación en Alimentación y Desarrollo A.C. Hermosillo, Sonora, México; Maestra en Ciencias de la Ingeniería Industrial (2013) por la Universidad de Sonora, México; Ingeniera Industrial en Electrónica (2005), por el Instituto Tecnológico Nacional de México, en Hermosillo, Sonora, México. Áreas de interés: Desarrollo humano, Desarrollo regional, Políticas públicas, Pobreza, Bienestar y Desigualdad, Metodologías de investigación. ORCID https://orcid.org/0000-0002-7704-260X.

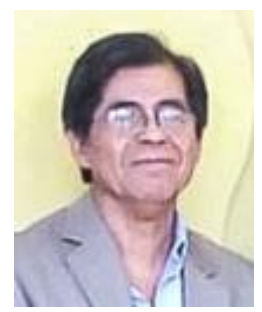

Mario Camberos Castro. Dr. en Ciencias Económicas, por la Universidad Autónoma de Baja California, Tijuana, México; Maestro en Ciencias Económicas por la Universidad Nacional Autónoma de México; Licenciado en Economía por la Universidad de Guadalajara, México; Investigador titular "E" en Centro de Investigación y Desarrollo A.C. (CIAD), Dpto. de Economía; Miembro del Sistema Nacional de Investigadores, Nivel II; Profesor investigador emérito del CIAD, Miembro de la Academia Mexicana de Ciencias (AMCE); Evaluador del Consejo Nacional para la Acreditación de las Ciencias Económicas. Líneas de investigación: Política económica, Bienestar, Desigualdad, Pobreza, Mercados laborales y Evaluación de Políticas Sociales. ORCID:https://orcid.org/0000-0002-3271-2980. 